\title{
Author Correction: Requirement for the basic helix-loop-helix transcription factor Dec2 in initial $\mathrm{T}_{\mathrm{H}} 2$ lineage commitment
}

Xuexian O. Yang, Pornpimon Angkasekwinai, Jinfang Zhu, Juan Peng, Zhiduo Liu, Roza Nurieva, Xikui Liu, Yeonseok Chung, Seon Hee Chang, Bing Sun and Chen Dong

Correction to: Nature Immunology https://doi.org/10.1038/ni.1821, published online 1 November 2009.

In the supplementary information originally posted for this article, in Supplementary Fig. 3, the flow cytometry plot in the spleen panel that is second from the left in the bottom row was incorrect. The correct plot is shown below. The error has not been corrected in the original article.

Original

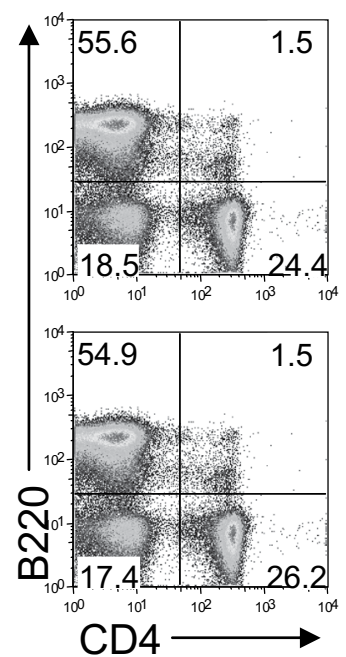

Corrected

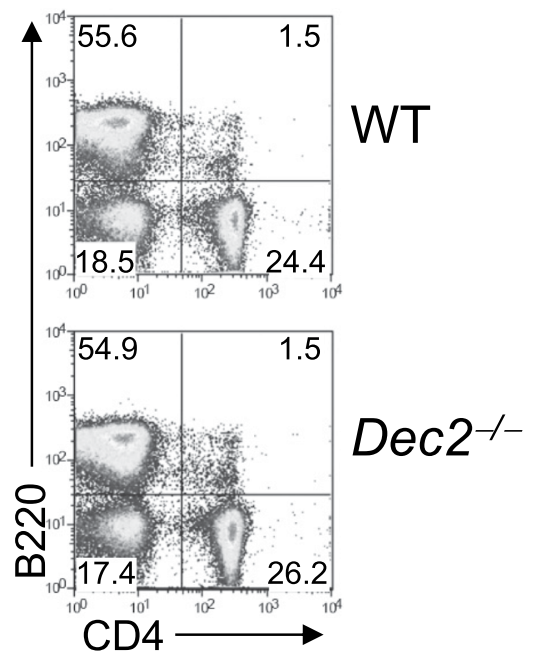

Supplementary Fig. 3 | Original and corrected.

\section{Additional information}

Supplementary information is available for this paper at https://doi.org/10.1038/s41590-020-0787-1.

Published online: 3 September 2020

https://doi.org/10.1038/s41590-020-0787-1

(C) The Author(s), under exclusive licence to Springer Nature America, Inc. 2020 